\title{
Brachial plexus injury presenting as focal myoclonus
}

\author{
Vijay Sardana ${ }^{1}$, Nishtha Jain,"* \\ ${ }^{1}$ Senior Professor \& HOD, ${ }^{2}$ Senior Resident, Dept. of Neurology, Government Medical College, Kota, Rajasthan, India
}

*Corresponding Author:

Email: docnishtha@gmail.com

\begin{abstract}
Myoclonus is characterised by quick, involuntary muscle jerks. It can be focal or generalized. It arises most commonly from the sensorimotor cortex, brainstem or the spinal cord. Rarely focal lesions of the peripheral nerves, plexus, or nerve roots may produce peripheral myoclonus. ${ }^{1}$ Here we report a case of 32 years old female who presented with focal myoclonus of the left upper limb secondary to brachial plexus injury and got treated successfully with botulinum toxin injection.
\end{abstract}

Keywords: Myoclonus, Focal Myoclonus, Brachial Plexus, Botulinum Toxin Injection.

\section{Introduction}

Myoclonus is usually of central origin. Impulses may be generated in the cerebral cortex or brainstem, or in the spinal cord with synchronous contractions in muscles innervated by one spinal segment (Segmental myoclonus). ${ }^{2}$ Recently, myoclonic-like movements have also been attributed to lesions in peripheral nerves and plexus. We present a patient, in whom rhythmic myoclonus clinically and electromyographically seemed to arise from a lesion in the posterior cord of the brachial plexus.

\section{Case Report}

A 32 years old female presented with chief complaints of abnormal flexing movements of the left upper limb at the elbow joint from last 10 days. She was having left frozen shoulder with severe pain and while examination when her arm was abducted she had severe pain along with onset of these abnormal jerking movements. These have been present since then, rhythmic in character and also observed in sleep though used to decrease in amplitude. The movements used to decrease in amplitude while holding the arm in flexed and adducted position and used to increase whenever extension and abduction movements were tried. There was no associated movements of the face and other limbs.

Neurological examination revealed myoclonus of the left arm at a rate of about $5 \mathrm{~Hz}$, both at rest and during arm movements. It was unaffected by other arm positions, sensory stimuli, or voluntary manoeuvers. Rest of the neurological examination was normal.

Routine haematological and biochemical tests were within normal limits. MRI Brain with cervical spine was normal. MRI Left brachial plexus showed altered signal intensity associated with thinning involving distal part of inferior trunk C6-C7 roots appearing hyper intense on Short tau inversion recovery (STIR)sequence and T2 weighted sequence suggesting possibility of Brachial Plexopathy. Electroencephalography (EEG) was normal. Electromyography (EMG) done using needle electrodes recorded rhythmic activity synchronous with jerky movements (maximum in the brachioradialis muscle and also in the biceps). Other muscles including deltoid, triceps and small muscles of hand were also sampled but showed no abnormal activity.

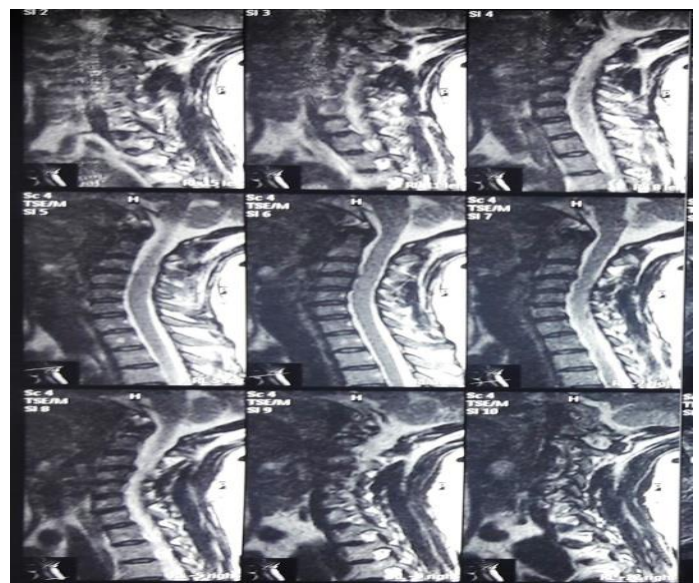

Fig. 1: T2 weighted Saggital image of cervical Spine (does not show any significant disc bulge or intensity changes in the spinal cord)

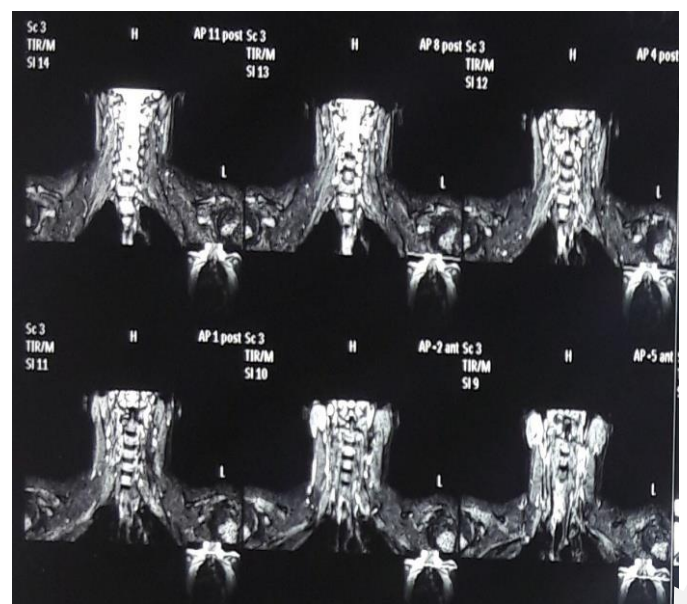

Fig. 2: MRI brachial plexus showing hyper intensity in distal part of inferior trunk on left side 


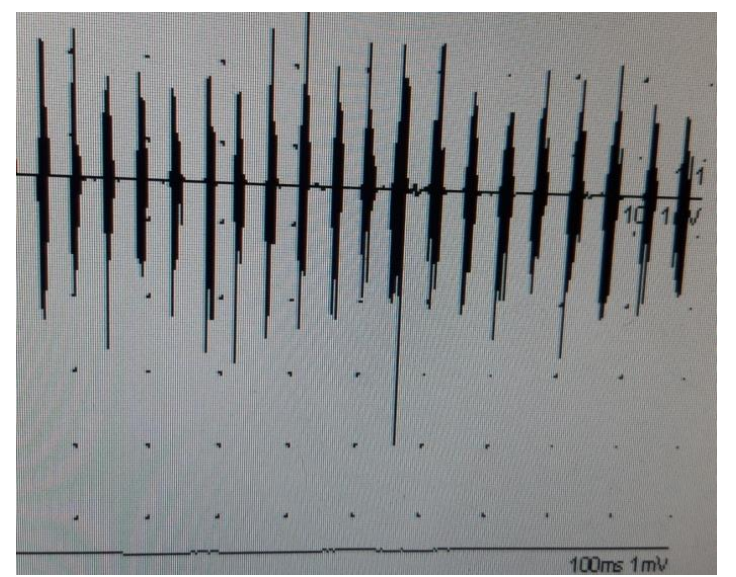

Fig. 3: EMG of Brachioradialis of left upper limb showing continuous rhythmic spontaneous activity synchronous with the limb movement.

In view of the clinical findings and findings on MRI Brachial plexus and EMG findings of the left upper limb possibility of peripheral myoclonus secondary to brachial plexus injury was kept and patients was started on benzodiazepines and sodium valproate. But there was no improvement in myoclonus. Patient was given 40 units of botulinum toxin in brachioradialis muscle of the left upper limb and 30 units in the biceps brachii muscle. After one week there was decrease in the amplitude of movements but were still persisted so second dose was planned and after 10 days of the first dose, second dose was given. This time 10 units each were given in two heads of biceps brachii and 10 units in brachioradialis Muscle. After one week of second dose, myoclonus completely stopped and patient was discharged.

\section{Discussion}

There is direct and indirect evidence to suggest that myoclonus in our patient was due to a peripheral nerve lesion. Evidently, the onset of myoclonus was associated with a traumatic event, a forceful and painful abduction of a "stiff' shoulder. The topography of EMG findings suggests that the generator is located in the C5-C6 segment of the brachial plexus.

Unlike irregular, epileptiform myoclonus of central origin, jerking in our patient was unenhanced by sensory stimuli, was present in sleep, and unaccompanied by EEG abnormalities or other evidence of cortical or brainstem involvement such as exaggeration on voluntary action, affection of speech or gait and startle response. A Spinal cord pathology seems unlikely, because of the absence of abnormal activity in muscles with the same segmental innervation as the involved muscles and absence of any radiological evidence of a lesion in the cervical spine or cord.

The treatment of myoclonus depends on the part of Neuraxis involved and the underlying Etiology. So it is important to establish the pathophysiology of myoclonus in a given patient because different drugs will work in different types of myoclonus. ${ }^{3}$ In general, antiepileptic agents such as Sodium Valproate, Piracetam and Leviteracetam are effective in cortical myoclonus but not in other types of myoclonus. Clonazepam can be helpful in other types of myoclonus also. Botulinum toxin injections have been used for palatal myoclonus and segmental spinal myoclonus with variable success. ${ }^{3,5}$ Botulinum toxin has been known to cause the blockage of the cholinergic transmission of the fusimotor fibres and also raise the level of excitability of the motor neurons. ${ }^{6}$ As our patient was not responding to the conventional drug treatment so she was given botulinum toxin therapy to which she responded well.

\section{Conclusion}

When a patient present with myoclonus, the first step is to localise the site of origin with history, Clinical examination and investigations. Brachial plexus injury is a rare cause of myoclonus. This case highlights high degree of suspicion for a peripheral cause of myoclonus which was not previously well recognised and importance of botulinum toxin therapy in effective management of such cases.

\section{References}

1. Caviness JN et al. The incidence and prevalence of myoclonus in Olmsted County, Minnesota. Mayo clin Proc 1999;74(6):565-69.

2. G Banks et al. Brachial plexus myoclonus. J Neurol Neurosurg Psychiatry 1985;48:582-84.

3. M M. Anis et al. Treatment of Palatal Myoclonus with Botulinum Toxin Injection. Case Reports in Otolaryngol 2013. Article ID 231505.

4. Kojovic et al. Myoclonic disorders: a practical approach for diagnosis and treatment. Ther Adv Neurol Disord 2011;4(1):47-62.

5. Jabbari B. History of botulinum toxin treatment in movement disorders. Tremor Other Hyperkinet Mov 2016;6.

6. Lagueny et al. Stimulus-Sensitive Spinal Segmental Myoclonus Improved With Injections of Botulinum Toxin Type A. Movement Dis 1999;14(1). 